Canadian Oncology

Nursing Journal

Revue canadienne

de soins infirmiers

en oncologie

Volume 30, Issue 4 • Fall 2020

elSSN: 2368-8076 


\title{
Le vécu des infirmières sur la sédation palliative pratiquée selon les recommandations suisses de 2005 : une étude exploratoire
}

\author{
par Martyna Tomczyk et Bernard N. Schumacher
}

\section{RÉSUMÉ}

De nombreuses études internationales ont démontré que le processus de la sédation palliative dans un contexte de fin de vie peut être mal vécu par les infirmières ${ }^{1}$, voire provoquer une détresse émotionnelle. Toutefois, le vécu des infirmières exerçant en soins palliatifs en Suisse n'a jamais été exploré, à notre connaissance. L'objectif de notre étude était donc de connaître et de décrire le vécu des infirmières relatif au processus de la sédation palliative basé sur les recommandations suisses de 2005. Afin d'atteindre cet objectif, une étude qualitative exploratoire monocentrique avec entretiens individuels compréhensifs a été privilégiée. Au total, dix infirmières ont été sollicitées et neuf ont effectivement participé. Après retranscription, huit entretiens ont été finalement inclus dans l'analyse. Cette dernière a montré que les infirmières vivaient le processus de la sédation palliative d'une manière méfiante, défiante ou encore confiante, ce qui était lié à la durée d'exercice en soins palliatifs. Ces résultats suggèrent que les recommandations suisses de 2005 ne protègent pas les infirmières contre l'incertitude liée au processus de la sédation palliative. Il s'avère donc nécessaire qu'une étude multicentrique nationale soit développée pour consolider ces résultats.

Mots-clés : sédation palliative, soins palliatifs, fin de vie, infirmières, vécu, étude qualitative.

\section{INTRODUCTION}

$\mathrm{L}$ a sédation au sens large du terme est une pratique imporLtante et indispensable dans le domaine des soins palliatifs (Cherny et ESMO Guidelines Working Group, 2014; Cherny et

1 Nous utilisons le féminin au sens générique pour ce mot tout au long de l'article.

\section{AUTEURS}

Martyna Tomczyk (auteure-ressource)

Chercheuse en éthique médicale, Ph.D., post-doctorante, Institut des humanités en médecine, CHUV et Université de Lausanne, Avenue de Provence 82, CH-1007 Lausanne, Suisse

martyna.tomczyk5891@gmail.com

Tél. : +33 (0)6 95118645

Bernard N. Schumacher, philosophe, professeur titulaire, Ph.D.-HDR, coordinateur de l'Institut interdisciplinaire d'éthique et des droits de l'homme, Université de Fribourg, Suisse.

Av. de Beauregard 13, CH-1700 Fribourg, Suisse

bernard.schumacher@unifr.ch

DOI: $10.5737 / 23688076304253260$ al., 2009). Toutefois, au plan épistémologique, elle ne bénéficie ni d'une terminologie univoque ni d'une définition consensuelle : divers termes et expressions sont utilisés pour la nommer qui en outre ne sont pas conceptualisés de la même manière, que ce soit dans la littérature médicale internationale (Papavasiliou, Payne et al., 2013; Papavasiliou, Brearley et al., 2013) ou dans les recommandations de bonnes pratiques professionnelles émises au niveau national ou européen (Abarshi et al., 2017; Gurschick et al., 2015; Schildmann et Schildmann, 2014).

En Suisse, le Groupe d'experts de la Société suisse de médecine et de soins palliatifs (2005) a publié des recommandations nationales en matière de sédation palliative. Ces experts y ont retenu l'expression « sédation palliative » et l'ont définie comme une « administration intentionnelle de substances sédatives au dosage minimal nécessaire dans le but d'obtenir le soulagement d'un ou plusieurs symptômes réfractaires en réduisant l'état de conscience temporairement ou définitivement d'un patient porteur d'une maladie avancée dont l'espérance de vie estimée est courte (jours ou semaines), ceci en collaboration avec une équipe multidisciplinaire compétente » (Groupe d'experts de la Société suisse de médecine et de soins palliatifs, 2005, p. 1). Dès lors, ces recommandations constituent un cadre de référence pour la sédation palliative dans ce pays. Afin de permettre leur application, divers protocoles et outils ont été développés localement, dans des institutions suisses offrant des soins palliatifs. À titre d'exemple, l'équipe de l'unité de soins palliatifs de l'Hôpital fribourgeois à Villarssur-Glâne dans le canton de Fribourg en Suisse romande a développé un outil de surveillance du patient, répondant aux besoins de l'équipe qui conduit le processus de la sédation palliative conformément aux recommandations suisses de 2005 (Francisco et Cantin, 2013).

S'il existe quelques études sur la sédation palliative en Suisse, elles s'appuient essentiellement sur des perspectives de médecins (Beauverd et al., 2014; Dumont et al., 2015; Foley et al., 2015; Ziegler et al., 2018). À ce jour, nous n'avons recensé, à travers des bases de données, qu'une seule étude abordant cette problématique d'un point de vue des infirmières (Tomczyk et Dieudonné-Rahm, 2019).

Les recommandations suisses (2005), comme plusieurs autres recommandations (Cherny et ESMO Guidelines Working Group, 2014; Cherny et al., 2009; Società Italiana di Cure Palliative, 2007; Société québécoise des médecins de soins palliatifs et Collège des médecins du Québec, 2016; Royal Dutch Medical Association, 2009) ne précisent pas spécifiquement le rôle des infirmières dans le processus de la 
sédation palliative. Or, ce rôle nous paraît essentiel, et ce à tout moment du processus. Par exemple, durant la phase initiale, les infirmières peuvent participer aux discussions en équipe, sans pour autant prendre la décision, cette dernière étant médicale. Par ailleurs, leur rôle est primordial dans la mise en œuvre de la sédation, la surveillance du patient, ainsi que l'évaluation de son confort. Cependant, une telle implication est susceptible d'être mal vécue par les infirmières, voire de provoquer une détresse émotionnelle, ce qui a été démontré par plusieurs études (Abarshi et al., 2014; Lokker et al., 2018; Zuleta-Benjumea et al., 2018). À ce jour², à notre connaissance, le vécu des infirmières exerçant en Suisse romande n'a pas été exploré dans le contexte du processus de la sédation palliative.

L'objectif de notre étude était donc de connaître et de décrire le vécu des infirmières d'une unité de soins palliatifs où la pratique de la sédation palliative est basée sur les recommandations suisses de 2005. La notion de vécu correspondait à l'« expérience vécue, réalité telle qu'elle a été vécue » (Centre national de ressources textuelles et lexicales, 2020), sachant qu'une telle expérience est tout naturellement empreinte de subjectivité.

\section{MATÉRIEL ET MÉTHODES}

Les informations sur le vécu des infirmières ont été extraites des entretiens réalisés lors d'une étude portant sur la conceptualisation de la sédation palliative et publiée antérieurement (Tomczyk et Dieudonné-Rahm, 2019). Elles en ont spontanément émergé et étaient les plus dominantes; il s'agit donc d'une analyse secondaire des données (Brugidou et al., 2005). Par conséquent, excepté le sous-paragraphe relatif à l'analyse des données, les autres sous-paragraphes ci-dessous se réfèrent à l'étude sur la conceptualisation (Tomczyk et Dieudonné-Rahm, 2019).

Il s'agissait d'une étude qualitative à visée exploratoire fondée sur des entretiens compréhensifs (Blanchet et Gotman, 2010) et réalisée conformément aux recommandations internationales des recherches qualitatives Consolidated Criteria for Reporting Qualitative Research - COREQ (Tong, Sainsbury, et Craig, 2007), sur une période de trois semaines, entre octobre et novembre 2017.

\section{a) Aspects législatifs et éthiques}

Conformément à la législation en vigueur en Suisse, cette étude non interventionnelle, ne comportant aucune récolte des données personnelles de santé et ne concernant pas directement les patients, n'exigeait pas l'avis du comité d'éthique. En revanche, l'autorisation du chef du service où se déroulait l'étude a été obtenue, et le consentement oral de chaque participante a été systématiquement recherché et obtenu dans tous les cas. De plus, l'autorisation d'enregistrer les entretiens à l'aide d'un dictaphone a été recueillie auprès de chaque participante. Afin que le chef de service et les participantes puissent donner leur consentement libre et éclairé, les informations suivantes leur ont été exposées : les objectifs de l'étude, la nature des informations à recueillir, le caractère de l'entretien (l'entretien

2 Novembre 2017, mars 2020. compréhensif supposant une analyse secondaire des données), le type de participation attendue (type de questions et durée approximative de l'entretien) et la raison de la demande de participation. Divers droits de participantes tels que le droit de refuser de participer à l'étude, le droit de ne pas répondre à des questions, le droit de mettre fin à l'entretien sans avoir à se justifier ont été rappelés. Il a été également mentionné que dans les publications issues de cette étude, aucune information permettant d'identifier les participantes ne serait indiquée. Enfin, il a été précisé que cette étude était menée dans le cadre d’une recherche postdoctorale de Martyna Tomczyk.

\section{b) Lieu d'étude}

Pour des raisons pratiques, l'unité stationnaire de soins palliatifs de l'Hôpital fribourgeois (HFR) située à Villars-surGlâne dans le canton de Fribourg en Suisse romande ${ }^{3}$ a été choisie comme terrain d'étude. Au moment de la réalisation de l'étude, cette unité possédait le label « qualitépalliative » attribué par l'Association suisse pour la qualité dans les soins palliatifs ${ }^{4}$ et comptait 14 lits spécialisés ainsi qu'un service d'accueil de jour.

\section{c) Critères d'inclusion}

Il y avait un seul critère d'inclusion : être un ou une infirmière acceptant de participer à l'étude et disponible; aucun critère restrictif n'étant imposé.

\section{d) Démarche d'inclusion}

Parmi l'équipe d'infirmières exerçant au sein du service, dix professionnels ont été aléatoirement choisis par leur responsable et invités à participer à un entretien individuel d'une vingtaine de minutes. Une inclusion progressive et espacée dans le temps a été privilégiée.

\section{e) Préparation des entretiens}

S'agissant des entretiens compréhensifs (Blanchet et Gotman, 2010), un guide d'entretien n'avait pas été élaboré au préalable, afin de «faire parler les informateurs autour du sujet, l'idéal étant de déclencher une dynamique de conversation » (Kaufmann, 2011). Par conséquent, une seule et même question a été posée à chaque participante : « Comment comprenez-vous l'expression "sédation palliative"? » Des informations concernant le vécu ont spontanément émergé lors des entretiens. Dans le souci et dans le souci d'obtenir des données fiables, des questions supplémentaires, initiées à partir du discours de l'interviewé, ont été posées.

\section{f) Réalisation des entretiens}

Les entretiens individuels compréhensifs ont été réalisés par la même personne - Martyna Tomczyk, post-doctorante en éthique médicale, expérimentée en recherche qualitative depuis huit ans au moment de la réalisation des entretiens. Elle n'avait aucun lien hiérarchique, familial ou autre ni avec le

3 L'anonymat concernant le lieu d'étude est levé sur l'autorisation du chef du service.

4 Association suisse pour la qualité dans les soins palliatifs : https://www.qualitepalliative.ch/association/?L=2 (Accès le 30 mars 2020). 
chef de service ni avec les participantes; il s'agissait d'une première rencontre dans tous les cas.

Tous les entretiens ont été réalisés à huis clos dans une pièce libre au sein du service (p. ex. salle de conférence, salle pour famille, etc.). La langue d'entretien était le français langue commune de tous les participantes, y compris de la personne réalisant ces entretiens (la langue maternelle première ou seconde ou encore une langue apprise; le canton de Fribourg étant un canton bilingue ${ }^{5}$ ). Tous les entretiens ont été enregistrés au moyen d'un dictaphone avec l'accord de chaque participante.

\section{g) Préparation des entretiens pour analyse}

Tous les entretiens ont été retranscrits manuellement et de manière quasi intégrale; les répétitions étant omises, afin de ne pas alourdir les données et par-là l'analyse. Les informations permettant d'identifier potentiellement les participantes ont été supprimées; seule la durée d'exercice en soins palliatifs étant gardée. Les entretiens retranscrits n'ont pas été envoyés aux participantes, pour relecture ou correction. Tous les enregistrements ont été supprimés une fois tous les entretiens retranscrits.

\section{h) Analyse des entretiens}

Une analyse thématique avec thématisation en continu a été privilégiée : les thèmes en lien avec le vécu des infirmières étaient identifiés et notés au fur et à mesure de la lecture de chaque entretien. Ensuite, ils ont été hiérarchisés sous la forme des thèmes centraux et de sous-thèmes auxquels des verbatim significatifs ont été associés. Enfin, les entretiens ont été analysés d'une manière transversale, en fonction des thèmes et des sous-thèmes préalablement identifiés, afin de faire émerger des points de convergence et de divergence. L'ensemble de l'analyse a été effectué manuellement par Martyna Tomczyk. Dans le souci de rigueur scientifique et afin de diminuer le biais de subjectivité, cette analyse a été refaite deux fois à un certain intervalle de temps par la même personne. Des ajustements issus d'une seconde et troisième analyse ont été intégrés. Les résultats issus d'une troisième analyse ont été revus par Bernard Schumacher.

\section{RÉSULTATS}

Dix infirmières ${ }^{6}$ ont été sollicitées pour participer à l'étude, dont neuf étaient disponibles et y ont effectivement participé. Le point de saturation a été observé autour du quatrième entretien; les entretiens suivants n'apportant plus de nouveaux éléments, et chaque entretien devenant de plus en plus redondant (Alami et al., 2009; Mucchieli, 2004; Van Campenhoudt et Quivy, 2011). Toutefois, l'étude a été poursuivie jusqu'au $9^{\mathrm{e}}$ entretien, par prudence et rigueur. Ainsi, neuf entretiens ont été réalisés et soumis à la retranscription. Cependant, lors de la retranscription, un entretien a dû être écarté, en raison d'une trop médiocre qualité de l'enregistrement. Au total, huit entretiens ont été inclus dans l'analyse.

5 Le français et l'allemand.

6 Afin de garder l'anonymat des participantes, les caractéristiques de ces dernières ne sont pas exposées ici.
L'entretien étant initialement prévu pour une vingtaine de minutes, cette durée était variable en pratique : 12 minutes pour le plus court et 52 minutes pour le plus long. La durée de tous les entretiens réunis était de 3 heures et 14 minutes.

Le vécu des infirmières interrogées s'est structuré autour d'un des trois axes principaux et indépendants : la méfiance, la défiance et la confiance.

\section{Méfiance}

La plupart des infirmières (6/8) vivaient le processus de la sédation palliative d'une manière méfiante, en se posant une question fondamentale sur le confort du patient.

\section{a) Le patient est-il réellement confortable et exempt de toute souf- france sous sédation?}

L'incertitude quant au confort du patient sous sédation palliative était la principale préoccupation rapportée par la plupart des infirmières (6/8). Elles l'ont expliqué ainsi :

«On place le patient dans le coma et après... nous, on se demande comment ils vivent l'expérience... C'est ça surtout. Pour moi, il dort, il est tranquille, mais je ne sais pas ce qui se passe réellement dans sa tête. [...] On ne sait pas comment la personne vit ce moment. C'est mon gros souci. [...] Il y a eu quelques sédations... pas beaucoup, mais il y en a eu quelques-unes... Pour eux, c'était juste insupportable... Ils vivaient très mal cette sédation, il $y$ avait des histoires de cauchemars. La personne était très contente qu'on ait levé la sédation et qu'elle ne soit plus enfermée dans ses cauchemars. Depuis, je me pose de plus en plus de questions. » (IDE $\left.n^{\circ} 1\right)$

Une infirmière $(1 / 6)$ a commencé à prendre conscience de cette incertitude au moment de l'entretien :

«La vie sous sédation, c'est une vie différente. Vous savez... Là, j’y réfléchis encore plus... Par exemple, quand je travaille la nuit, je fais des tournées régulièrement, je vois des malades qui dorment bien. Enfin, moi, j'ai l'impression qu'ils dorment bien. Et le matin, certains d'entre eux me disent qu'ils ont passé une mauvaise nuit. Décidément, il y a une part de mystère entre ce qu'on observe et ce que les malades vivent dans le sommeil... (sommeil naturel ou sommeil artificiel). Après, justement, il faut se poser la question : est-ce que le sommeil sous sédation diffère d'un sommeil normal? Enfin, je voudrais vous dire qu'on ne peut jamais totalement savoir ce qui se passe dans la tête des malades sédatés... " (IDE $n^{\circ}$ 2)

\section{b) Éléments permettant de nuancer la méfiance des infirmières}

Les deux principaux éléments permettant de nuancer la méfiance des infirmières ont été identifiés. Il s'agit d'une évaluation approfondie de la situation du patient par l'équipe de soins, ainsi que la possibilité et la prise en compte de l'évaluation par le patient lui-même lorsque la sédation est levée.

- Évaluation du confort du patient sédaté par l'équipe de soins (6/6):

"Moi, quand j'évalue la sédation, je regarde toujours le visage. Il y a une part de mystères; quand quelqu'un est sédaté... Il $y$ a des signes cliniques qu'on ne peut pas observer, 
mais le visage... le visage, c'est spécial... C'est vraiment particulier. S'il $y$ a une souffrance, ça se voit tout de suite sur le visage, même si le malade est endormi. Je ne sais pas comment vous expliquer tout cela... C'est assez mystérieux... Ça se voit toujours sur le visage. [...] Par exemple, le patient fait des grimaces, crispe le visage... C'est un signe pour nous qu'il $y$ a une souffrance. » (IDE $\left.n^{\circ} 2\right)$

- Évaluation du confort par le patient lui-même, une fois la sédation levée $(6 / 6)$ :

"Personnellement, je suis très rassurée quand il $y$ a cette levée de sédation, que la personne puisse nous dire comment elle a vécu les choses. [...] Une fois la sédation levée, on demande au patient comment il se sent, comment il a vécu la sédation. Pour moi, c'est très important. On peut avoir un retour, on peut échanger. On a des patients qui voulaient vraiment la sédation, qui étaient dans une grande souffrance et quand on a levé la sédation, ils ne voulaient plus qu'on reprenne; ils n'étaient pas bien sous cette sédation, ils étaient déconnectés de la famille, alors que pour eux, c'était vraiment très important d'avoir cet échange. Je trouve que c'est très important qu'ils puissent dire leur vécu. » (IDE $\left.n^{\circ} 4\right)$

En résumé, la principale source de méfiance vis-à-vis de la sédation était le confort du patient. Lévaluation du confort, que ce soit par l'équipe de soins quand le patient était sous sédation ou par le patient lui-même quand la sédation a été levée, était de nature à diminuer cette méfiance. Lévaluation du confort par le patient lui-même était d'autant plus rassurante qu'elle permettait d'éliminer la part subjective de l'évaluation faite par les infirmières.

\section{Défiance}

Une infirmière $(1 / 8)$, dont la durée d'exercice en soins palliatifs était la plus longue, vivait le processus de la sédation palliative d'une manière défiante.

\section{a) Est-il opportun de mettre en place une sédation palliative sachant qu'elle ne soulage pas la souffrance du patient?}

La certitude quant au non-soulagement de la souffrance du patient à l'aide d'une sédation palliative était la principale préoccupation rapportée par une infirmière (1/8). Elle l'a expliqué ainsi :

«Ce n'est pas parce qu'on voit quelqu'un qui ne bouge plus, qui ne réagit plus, qu'il ne souffre pas. Déjà, s'il $\gamma$ a des patients à qui on lève la sédation et qui nous disent qu'ils n'étaient pas bien... Ça donne à penser... Pour moi, c'est suffisant! C'est très bien les publications des médecins. Moi, je préfêre entendre les ressentis des malades, au lieu de lire les publications des médecins. [...] Personnellement, je pense que ça ne soulage pas la souffrance. Pour moi, la souffrance et la sédation... Moi... je ne peux jamais promettre à personne qu'il ne souffrira pas pendant la sédation... Jamais! [...] Je pense que la souffrance, ça fait partie de notre condition humaine et on ne peut pas tout résoudre grâce à une substance médicamenteuse prescrite par les médecins. » (IDE $\left.n^{\circ} 8\right)$
Pour élaborer ses propos, elle a évoqué une situation clinique :

«Je me souviens encore d'une situation (ce n'était pas ici). Le patient était confus, dans une grande souffrance. Je pense qu'il revivait des choses du passé. Le médecin voulait qu’on lui fasse la sédation. Moi, j’ai dit: "Non!" Je suis resté avec ce monsieur. Il s'est calmé et il m'a raconté plein de choses. On a discuté et discuté et puis, il s'est endormi. Il a passé une belle nuit. Deux jours plus tard, quand je suis retournée au boulot, le monsieur était sous sédation et c'était jusqu'au bout. Il est décédé trois jours après... Voilà. Pour moi, la sédation, c'est plutôt un évitement. On évite d'affronter la souffrance du patient. On la fait taire. Et c'est ça qui me gêne. » (IDE n 8)

Plus encore, cette infirmière a évoqué qu'une sédation palliative risque même de renforcer la souffrance :

"Il ne faut pas non plus oublier que la sédation, c'est parfois pire que la souffrance elle-même. Je me souviens d'une patiente qui était sous sédation, puis la sédation a été levée... Elle m'a dit qu'elle allait atrocement mal pendant cette sédation et elle ne voulait pas qu'on continue. Donc, moi, je suis sceptique... Je dis toujours aux médecins: "Attention, ne vous prenez pas pour Dieu”. On a des outils. Mais je pense qu'il faut avoir l'honnêteté de se dire: "On ne sait pas tout faire, on ne peut supprimer toute souffrance!” » (IDE $\left.n^{\circ} 8\right)$

\section{b) Éléments permettant de nuancer la défiance de l'infirmière}

En raison de sa défiance, cette infirmière préférait la présence auprès du patient à la mise en œuvre d'une sédation palliative :

"Quand on est auprès du malade, le malade s'ouvre... Il commence à expliquer ses angoisses. [...] Si on ne peut plus rien faire sur le plan technique, il faut simplement rester auprès de cette personne. Parfois, le fait d'accompagner cette personne soulage l'angoisse. Ça, j'ai vu ça plusieurs fois... mais vous savez la présence, ça prend du temps... » (IDE $\left.n^{\circ} 8\right)$

\section{Confiance}

Une infirmière $(1 / 8)$, dont la durée d'exercice en soins palliatifs était la plus courte, vivait le processus de la sédation palliative d'une manière confiante.

\section{a) La sédation palliative vécue d'une manière confiante}

Une infirmière a rapporté une expérience positive de la pratique de la sédation palliative et conforme à ses valeurs de soin. Contrairement à ses collègues interrogées (7/8), elle n'a pas explicitement abordé la question du soulagement de la souffrance du patient. Voici son témoignage :

"Moi, je n'ai pas le sentiment de faire quelque chose... les sédations que j'ai vécues, c'était vraiment un soulagement pour moi. Pour moi, c'est tellement fait dans le respect $d u$ patient. » (IDE $\left.n^{\circ} 6\right)$

\section{b) Principaux éléments contribuant à la confiance face à la pra- tique de la sédation}

Deux éléments étaient primordiaux dans la construction d'une expérience positive de la sédation chez cette infirmière : 
- Évaluation du confort du patient pendant la sédation, par l'équipe de soins :

«Aussi, l'évaluation, c'est fait par nous [...] Il faut toujours

évaluer selon notre grille. » (IDE $n^{\circ}$ 6)

- Évaluation du confort par le patient lui-même, une fois la sédation levée :

"Il y a quelque chose qui m'aide personnellement, c'est la levée de la sédation. Au bout de 24 heures, on lève la sédation et on essaie de voir le malade. [...] On cherche toujours avec cette grille, qui est vraiment un soutien pour nous. [...] La levée de la sédation nous permet de voir si cette sédation est efficace. On discute avec le patient. Parfois, cette première sédation fait baisser l'angoisse et puis on n'a pas besoin de reprendre la sédation. Dans ce contexte, c'est quelque chose de positif. (IDE $n^{\circ}$ 6)

En guise de résumé de tous les résultats, la plupart des infirmières éprouvaient une certaine méfiance (6/8), voire une défiance $(1 / 8)$, à l'égard du processus de la sédation palliative. La méfiance comme la défiance résultait essentiellement d'une incertitude relative au confort du patient. Si la méfiance était susceptible d'être nuancée, la défiance, quant à elle, paraissait inatténuable. A contrario, une infirmière vivait ce processus de manière plutôt confiante, et cette confiance pouvait même être renforcée.

Les résultats présentés ci-dessus sont résumés dans la figure 1.

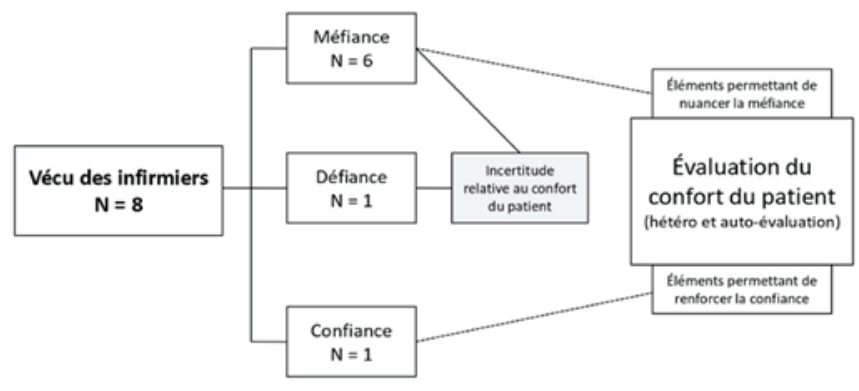

Figure 1 : Récapitulatif des résultats

\section{DISCUSSION}

Notre étude qualitative à visée exploratoire mettant en lumière le vécu des infirmières en lien avec la sédation palliative pratiquée selon les recommandations nationales en la matière, en Suisse romande, revêt un caractère original. Elle a essentiellement mis en évidence trois catégories de vécu des infirmières, dont l'une - celle focalisée sur la méfiance - fut dominante. En outre, elle a mis en relief un lien entre le vécu et la durée d'exercice en soins palliatifs : les infirmières plus expérimentées vivaient le processus de la sédation d'une manière méfiante, voire défiante, tandis que l'infirmière dont la durée d'exercice en soins palliatifs était la plus courte le vivait d'une manière plutôt confiante. L'évaluation du confort du patient par l'équipe de soins ainsi que par le patient lui-même était rassurante pour tous les participants, sauf l'infirmière défiante.
Plus spécifiquement, la plupart des infirmières étaient méfiantes, dont une défiante, à l'égard de la sédation palliative, en raison d'une incertitude quant au soulagement de la souffrance du patient. En effet, si l'objectif de la sédation palliative est de soulager un ou plusieurs symptômes réfractaires en réduisant l'état de conscience (Groupe d'experts de la Société suisse de médecine et de soins palliatifs, 2005), force est toutefois de constater qu'elle n'est pas un traitement ciblé ayant pour objectif de supprimer ce(s) symptôme(s) en agissant par un moyen pharmacologique sur des mécanismes physiopathologiques, mais plutôt un traitement permettant de diminuer ou de faire disparaître la perception de ce(s) symptôme(s) par l'altération de la vigilance (Davis, 2009a). Si les professionnels de santé ont recours à des échelles d'observation clinique telles que la Critical Care Pain Observational Tool, la Behavioral Pain Scale ou encore l'Échelle comportementale de la douleur chez la personne âgée durant les soins, ces outils présentent certaines limites. Développées dans d'autres contextes et souvent non validées dans cette situation particulière, ces échelles sont par ailleurs basées sur l'observation qui, par définition, est empreinte de subjectivité et, de ce fait, susceptible d'interprétation (Brinkkemper, van Norel, Szadek et al., 2013 ; Pudas-Tähkä, Axelin, Aantaa, Lund, et Salanterä, 2009). Il est en effet démontré que les évaluations faites par les médecins diffèrent de celles faites par les infirmières, et que des facteurs non cliniques, comme la religion ou l'âge du soignant, peuvent influencer cette évaluation (Demertzi, Schnakers, Ledoux et al., 2009).

De plus, s'il existe des échelles pour l'évaluation de la douleur ou de la dyspnée, aussi subjectives soient-elles, celles qui permettraient d'évaluer d'autres symptômes physiques (p. ex. les nausées) et surtout la souffrance psychique, existentielle ou morale ${ }^{7}$ font défaut. Or, l'altération de la vigilance, induite par la sédation, n'élimine pas l'activité cérébrale, ce qui permet potentiellement une poursuite des phénomènes psychiques comme les angoisses, les cauchemars, ou encore la perception de l'environnement. Si des travaux scientifiques relatifs à ce sujet font défaut dans le champ des soins palliatifs (Davis, 2009b; Kon, 2011), il en existe dans le domaine de l'anesthésie et de la réanimation (Ghoneim, 2007; Ghoneim et al., 2009; Leslie, Chan, Myles et al., 2010; Sanders et al., 2012). Il est intéressant de remarquer que, malgré ses limites, l'évaluation était mise en avant par les infirmières interrogées dans le cadre de notre étude comme un élément rassurant lors du processus de la sédation palliative.

Il convient aussi de souligner que ces infirmières interrogées attachaient une grande importance au visage du patient, lors de l'évaluation de son confort, tout en soulignant leurs difficultés à argumenter scientifiquement une telle démarche. Afin d'enrichir notre discussion dans une approche interdisciplinaire, nous proposons ici d'apporter des éléments de réflexion en se référant à la philosophie. À titre d'exemple, Emmanuel Levinas souligne que le

7 Les différences potentielles entre les trois concepts en question dépassant l'objet de notre article, elles ne sont pas développées ici. 
visage ne se laisse pas réduire à un « assemblage d'un nez, d'un front, d'yeux, etc. » (Levinas, 1995, p. 20), mais il renvoie à une dimension qui transcende l'idée que l'on se fait de l'autre. Le visage « n'est pas "vu”. Il est ce qui ne peut devenir un contenu » (Levinas, 2009, p. 81), menant toujours vers un au-delà qui échappe à l'emprise de la connaissance. Le visage se donne et en même temps se dérobe à toute tentative d'emprise. L'autre, souligne Levinas, « prend la signification d'un visage par la dimension nouvelle qu'il ouvre dans la perception d'un être. Par le visage, l'être n'est pas seulement enfermé dans sa forme et offert à la main - il est ouvert, s'installe en profondeur et, dans cette ouverture, se présente en quelque manière personnellement. »(Levinas, 2009, p. 20). Cette relation à autrui s'exprime de manière toute particulière lorsqu'une personne se trouve en état de solitude de par la souffrance et la mort : « Seul un être arrivé à la crispation de sa solitude par la souffrance et à la relation avec la mort, se place sur un terrain où la relation avec l'autre devient possible. » (Levinas, 1983, p. 64). Le visage qui ne cesse de parler en appelle à une réponse de la part du soignant, réponse qui est de l'ordre d'une responsabilité inconditionnelle envers autrui : « répondre à lui et déjà répondre de lui » (Levinas, 2009, p. 83; Pierron et al., 2019).

Lévaluation faite par le patient lui-même reste à ce jour une méthode fiable. S'il est tout naturellement impossible d'y avoir recours en cas de sédation profonde et continue jusqu'au décès, il est cependant possible d'y accéder en cas de sédation temporaire; une fois la sédation stoppée, le patient peut exprimer son vécu. Une telle démarche, parfaitement conforme aux recommandations suisses de 2005, était en effet rassurante pour toutes les infirmières sauf une. Il convient toutefois de souligner que si une sédation profonde et continue jusqu'au décès n'est explicitement préconisée en première intention par les experts suisses dans ce document national, il en va autrement au niveau européen; les experts européens recommandent en effet le recours à une telle sédation dans certaines situations bien précises (Cherny et al., 2009).

Reste enfin à discuter du vécu d'une infirmière qui, contrairement à ses collègues, vivait le processus de la sédation palliative d'une manière défiante. Considérant cette pratique comme étant inefficace contre la souffrance, voire l'intensifiant, elle estimait par ailleurs qu'elle constitue une entrave à une véritable relation de soin, la seule étant susceptible de soulager la souffrance du patient, selon cette infirmière. Tout d'abord, il convient de souligner que si « la relation entre hommes » est, comme le remarque Levinas, « le non synthétisable par excellence » (Levinas, 2009, p. 71), il est cependant certain que « la véritable union ou le véritable ensemble n'est pas un ensemble de synthèse, mais un ensemble de face à face. » (Levinas, 2009, p. 72). Or, la relation entre le professionnel de santé dont « la puissance d'agir est, au départ, plus grande que celle de son autre » (Ricœur, 1990, p. 255) et l'autre souffrant - vulnérable et fragile - est d'emblée une relation asymétrique, d'où l'exigence d'y insérer une certaine réciprocité. Paul Ricœur remarque en effet que « la réciprocité [...] se détache sur le fond de la présupposition d'une dissymétrie initiale entre les protagonistes de l'action - dissymétrie qui place l'un dans la position d'agent et l'autre dans celle de patient » (Ricœur, 1990, p. 255). Si l'être humain est « un être de réciprocité » (Malherbe, 1997, p. 26), cette réciprocité fondamentale manifeste « la coresponsabilité des êtres humains, tout un chacun censé tenir parole les uns par rapport aux autres » (Malherbe, 1997, p. 29). Pour ce philosophe, la parole est en effet « à la fois le but, le lieu et le moyen de la réciprocité » (Malherbe, 1997, p. 26), ce qui concorde avec les propos de l'infirmière interrogée dans le cadre de notre étude. Cette dernière soulignait en effet qu'une telle relation fondée sur la parole est susceptible de soulager la souffrance du patient. Ces propos paraissent d'autant plus intéressants que la souffrance est une expérience globale, plutôt existentielle, en lien avec une douleur ressentie dans le corps ou indépendamment de celle-ci (Perrot, 2016). Il y a quelques décennies, Cicely Saunders (1993), pionnière du mouvement des soins palliatifs, a déjà remarqué qu'une présence et une écoute attentives dans une ambiance bienveillante sont susceptibles d'apaiser la douleur physique. Inversement, un analgésique puissant risque de s'avérer inefficace, si la souffrance (douleur psychique) n'est pas soulagée. À cet égard, Verspieren (1984) affirme que le meilleur remède contre la souffrance est « une relation simple, vraie et profonde avec le malade » (p. 111). Si une telle relation est effectivement susceptible d'être un soin à part entière, elle est, comme l'explique Aubry, « un soin très difficile parce qu'il n'y a pas à proprement parler de savoirs sur ce qui fait souffrance » (Aubry, 2011, p. 230).

Avant de terminer, il convient de s'arrêter sur quatre principales limites méthodologiques de notre étude. Tout d'abord, il paraît légitime de se demander si le fait d'extraire des informations sur le vécu à partir des données d'une étude dont l'objectif premier était de découvrir et de décrire la conceptualisation de la sédation palliative par les infirmières est méthodologiquement acceptable; l'étude n'abordant pas ouvertement la question du vécu. Pour y répondre, il est nécessaire de rappeler que l'objectif de la recherche sur la conceptualisation à visée exploratoire et fondée sur des entretiens compréhensifs n'était pas d'expliquer un phénomène bien précis, mais plutôt de nous engager sur le terrain afin de faire apparaître les différentes facettes d'une réalité fort complexe (Alami et al., 2009; Paillé et Mucchielli, 2012). Le vécu des infirmières fut la facette dominante. Ensuite, l'absence de guide d'entretien peut être légitimement remise en question. En effet, nous avons choisi de ne pas l'élaborer afin que l'entretien reste le plus ouvert possible et fasse émerger le maximum de catégories. Une telle démarche est tout à fait conforme aux principes d'une recherche qualitative, et d'un entretien compréhensif en particulier (Kaufmann, 2011). Enfin, le nombre de participants est susceptible d'être questionné, alors qu'il correspond au principe de l'étude qualitative à visée exploratoire; dans ce type de recherche, il ne s'agit pas de la représentativité de la population, mais de sa « significativité sociale » (Alami et al., 2009). Plus spécifiquement, l'objectif de notre étude n'était pas de savoir combien d'infirmières ont tel ou tel vécu du processus de la sédation palliative, mais de faire émerger des 
catégories «transversales », pour explorer la population étudiée, via l'individualité de chaque participante.

Il convient aussi de préciser que les entretiens retranscrits n'ont pas été envoyés aux participantes pour relecture ou correction; ils ont été directement soumis à des analyses. Une telle démarche fut en cohérence avec l'objectif de notre étude principale (Tomczyk et Dieudonné-Rahm, 2019) qui consistait à recueillir les conceptualisations de chaque infirmière à un moment donné, c'est-à-dire lors de l'entretien, par association mentale et en lien avec ses expériences acquises jusqu’à ce moment-là; l'objectif n'étant ni de vérifier les connaissances théoriques ou pratiques sur la sédation palliative ni d'évaluer les pratiques au sein du service. Dans ce cas, la correction des entretiens par les participantes serait à l'encontre de l'objectif de l'étude et susceptible de biaiser les données et, par-là, les résultats. S'agissant d'une étude portant sur le vécu, il est en revanche préférable que les entretiens retranscrits soient envoyés aux participants (Ribau et al., 2005). Or, notre étude sur le vécu résultant d'une analyse secondaire des données, il nous fut impossible à cette étape-là de contacter les participantes, en raison de l'anonymisation des entretiens, ce qui peut potentiellement constituer une limite méthodologique.

\section{RÉFÉRENCES}

Abarshi, E. A., Papavasiliou, E. S., Preston, N., Brown, J., \& Payne, S. (2014). The complexity of nurses' attitudes and practice of sedation at the end of life: a systematic literature review. Journal of Pain and Symptom Management, 47(5), 915-25.e11. https://doi. org/10.1016/j.jpainsymman.2013.06.011.

Abarshi, E., Rietjens, J., Robijn, L. et al. (2017). International variations in clinical practice guidelines for palliative sedation: A systematic review. BMJ Supportive \& Palliative Care, 7(3), 223-9. https://doi. org/10.1136/bmjspcare-2016-001159.

Alami, S., Desjeux, D., \& Garabuau-Moussaoui, I. (2009). Les méthodes qualitatives. PUF.

Aubry, R. (2011). Réflexions sur les pratiques et le quotidien des médecins, sur le soin palliatif et le rapport à la mort dans une maladie incurable et douloureuse. Dans : Ferry, J.M, directeur, Fin(s) de vie : le débat. PUF, p. 217-241.

Beauverd, M., Bernard, M., Currat, T. et al. (2014). French Swiss physicians' attitude toward palliative sedation: Influence of prognosis and type of suffering. Palliative \& Supportive Care, 12(5), 345-50. https://doi.org/10.1017/S1478951513000278.

Blanchet, A., \& Gotman, A. (2010). L'enquête et ses méthodes : l'entretien. Armand Colin.

Brinkkemper, T., van Norel, A. M., Szadek, K. M. et al. (2013). The use of observational scales to monitor symptom control and depth of sedation in patients requiring palliative sedation: A systematic review. Palliative Medicine, 27(1), 54-67. https://doi. org/10.1177/0269216311425421.

Brugidou, M., Le Roux, D., \& Salomon, A. C. (2005). Colloque «L'analyse secondaire en recherche qualitative », Grenoble. https:// reanalyse.hypotheses.org/files/2014/09/Pr\%C3\%A9sentation.pdf (Accès le 04 avril 2020).

Centre National de Ressources Textuelles et Lexicales. Consulté sur : http://www.cnrtl.fr/definition/conceptualisation/substantif. (Accès le 04 avril 2020).

\section{CONCLUSION}

Cette étude a permis d'appréhender le vécu des infirmières en lien avec la pratique de la sédation palliative fondée sur les recommandations suisses de 2005 et avec un protocole facilitant leur application. Elle a fait émerger des catégories qui restent à consolider et compléter via d'autres études. Au-delà du vécu des infirmières, il serait essentiel d'explorer le vécu des patients ayant bénéficié d'une sédation palliative temporaire. Les données de ces futures études pourraient être utiles pour actualiser les recommandations en matière de sédation palliative et les protocoles institutionnels, dans le souci d'assurer les meilleurs soins aux patients en fin de vie et de prévenir le risque de détresse émotionnelle chez les infirmières.

\section{DÉCLARATION DE CONFLITS D'INTÉRÊTS}

Les auteurs déclarent n'avoir aucun conflit ni lien d'intérêt avec le présent article.

\section{REMERCIEMENTS}

Les auteurs remercient vivement le Dr Boris Cantin, chef du service de soins palliatifs à la Villa St. François de l'Hôpital fribourgeois, et Mme Sylvie Francisco, infirmière responsable, d'avoir rendu possible la réalisation de la présente étude. Ils sont également reconnaissants envers les infirmières ayant accepté d'y participer.

Cherny, N. I., Radbruch, L., \& The Board of the European Association for Palliative Care. (2009). European Association for Palliative Care (EAPC) recommended framework for the use of sedation in palliative care. Palliative Medicine, 23(7), 581-93. https://doi. org/10.1177/0269216309107024.

Cherny, N. I., \& ESMO Guidelines Working Group. (2014). ESMO Clinical Practice Guidelines for the management of refractory symptoms at the end of life and the use of palliative sedation. Annals of Oncology, (Suppl. 3), iii143-52. https://doi.org/10.1093/ annonc/mdu238.

Davis, M. (2009a). Palliative sedation: Four questions. Progress in Palliative Care, 17, 203-4.

Davis, M. (2009b). Does palliative sedation always relieve symptoms? Journal of Palliative Medicine, 12(10), 875-7. https://doi.org/10.1089/ jpm.2009.0148.

Demertzi, A., Schnakers, C., Ledoux, D. et al. (2009). Different beliefs about pain perception in the vegetative and minimally conscious states: A European survey of medical and paramedical professionals. Progress in Brain Research, 177, 329-38. https://doi. org/10.1016/S0079-6123(09)17722-1.

Dumont, S., Blondeau, D., Turcotte, V. et al. (2015). The use of palliative sedation: A comparison of attitudes of French-speaking physicians from Quebec and Switzerland. Palliative \& Supportive Care, 13(4), 839-47. https://doi.org/10.1017/S1478951514000364.

Francisco, S., \& Cantin, B. (2013). Suivi de la personne recevant une sédation palliative : quelles responsabilités pour l'infirmière? Revue de la Société suisse de médecine et de soins palliatifs « palliative ch », $4:$ 47-50. https://www.palliative.ch/fileadmin/user_upload/ palliative/magazin/2013_4.pdf

Foley, R. A., Johnson, W. S., Bernard, M. et al. (2015). Attitudes regarding palliative sedation and death hastening among swiss physicians: A contextually sensitive approach. Death Studies, 39(8), 473-82. https://doi.org/10.1080/07481187.2015.1029142. 
Ghoneim, M. M. (2007). Incidence of and risk factors for awareness during anaesthesia. Best Practice o Research Clinical Anaesthesiology, 21(3), 327-43. https://doi.org/10.1016/j.bpa.2007.05.002.

Ghoneim, M. M., Block, R. I., Haffarnan, M., \& Mathews, M. J. (2009). Awareness during anesthesia: Risk factors, causes and sequelae. A review of reported cases in the literature. Anesthesia Q Analgesia, 108(2), 527-35. https://doi.org/10.1213/ane.0b013e318193c634.

Groupe d'experts de la Société suisse de médecine et de soins palliatifs. (2005). Recommandations « Sédation palliative ». Consensus sur la meilleure pratique en soins palliatifs en Suisse. Bigorio. https:// www.palliative.ch/fileadmin/user_upload/palliative/fachwelt/E_ Standards/E_12_4_bigorio_2005_Sedation_fr._pdf.pdf

Gurschick, L., Mayer, D. K., \& Hanson, L. C. (2015). Palliative sedation: an analysis of international guidelines and position statements. American journal of hospice \& palliative care, 32(6), 660-71. https:// doi.org/10.1177/1049909114533002.

Kaufmann, J. C. (2011). L'enquête et ses méthodes. L'entretien compréhensif. Armand Colin.

Kon, A. (2011). Palliative sedation: It's not a panacea. American Journal of Bioethics, 11(6), 41-2. https://doi.org/10.1080/15265161.2011.5775 13.

Leslie, K., Chan, M. T., Myles, P. S., Forbes, A., \& McCulloch, T. J. (2010). Posttraumatic stress disorder in aware patients from the B-Aware trial. Anesthesia \& Analgesia, 110(3), 823-8. https://doi. org/10.1213/ANE.0b013e3181b8b6ca.

Levinas, E. (1983). Le temps et l'autre. PUF.

Levinas, E. (1995). Difficile liberté. Le Livre de Poche.

Levinas, E. (2009). Éthique et infini. Le Livre de Poche.

Lokker, M. E., Swart, S. J., Rietjens, J. A. C., van Zuylen, L., Perez, R. S. G. M., van der Heide, A. (2018). Palliative sedation and moral distress: A qualitative study of nurses. Applied Nursing Research, 40, 157-61. https://doi.org/10.1016/j.apnr.2018.02.002.

Malherbe, J. F. (1997). Pour une éthique de la médecine. Artel-Érasme.

Mucchieli, A. (2004). Dictionnaire des méthodes qualitatives en sciences humaines. Armand Colin.

Paillé, P., \& Mucchielli, A. (2012). L'analyse qualitative en sciences humaines et sociales. Armand Colin.

Papavasiliou, E., Brearley, S. G., Seymour, J. E., Brown, J., Payne, S. A.; EURO IMPACT. (2013). From sedation to continuous sedation until death: How has the conceptual basis of sedation in end-of-lifecare changed over time? Journal of Pain and Symptom Management, 46(5), 691-706. https://doi.org/10.1016/j.jpainsymman.2012.11.008.

Papavasiliou, E., Payne, S., Brearley, S., Brown, J., \& Seymour, J. (2013). Continuous sedation (CS) until death: Mapping the literature by bibliometric analysis. Journal of Pain and Symptom Management, 45(6), 1073-82.e10. https://doi.org/10.1016/j. jpainsymman.2012.05.012.

Perrot, S. (2016). Les bases neurophysiologiques de la douleur. Dans : Perrot, S. Douleur, soins palliatifs et accompagnement. Éditions MedLine, p. 17-30.

Pierron, J.Ph., Schumacher, B., \& Zielinski, A. (2019). Levinas et le soin. Numéro spécial (2019-1, nr. 14) de Ethique, politique, religions. Paris: Classiques Garnier.
Pudas-Tähkä, S. M., Axelin, A., Aantaa, R., Lund, V., \& Salanterä, S. (2009). Pain assessment tools for unconscious or sedated intensive care patients: a systematic review. Journal of Advanced Nursing, 65(5), 946-56. https://doi.org/10.1111/j.1365-2648.2008.04947.x.

Ribau, C., Lasry, J. C., Bouchard, L., Moutel, G., Hervé, C., \& MarcVergnes, J. P. (2005). La phénoménologie : une approche scientifique aux expériences vécues. Recherche en soins infirmiers, 81(2), 21-7. https://doi.org/10.3917/rsi.081.0021.

Ricœur, P. (1990). Soi-même comme un autre. Éditions du Seuil.

Royal Dutch Medical Association (KNMG). (2009). Guideline for Palliative Sedation Royal Dutch Medical Association. https:// palliativedrugs.com/download/091110_KNMG_Guideline_for_ Palliative_sedation_2009_2_\%5B1\%5D.pdf.

Sanders, R., Tononi, G., Laureys, S., Sleigh, J., \& Stat, D. (2012). Unresponsiveness $\neq$ Unconsciousness. Anesthesiology, 116(4), 94659. https://doi.org/10.1097/ALN.0b013e318249d0a7.

Saunders, C. (1993). Some challenges that face us. Second Congress of the European Association for Palliative Care Brussels, 19-22 October, 1992. Palliative Medicine, 7, 77-83.

Schildmann, E., \& Schildmann J. (2014). Palliative sedation therapy: A systematic literature review and critical appraisal of available guidance on indication and decision-making. Journal of Palliative Medicine, 17, 601-612.

Società Italiana di Cure Palliative. (2007). Raccomandazioni della SICP sulla sedazione terminale/ sedazione palliative. https://www. fondazioneluvi.org/wp-content/uploads/2017/01/SedazioneTerminale-Sedazione-Palliativa.pdf

Société québécoise des médecins de soins palliatifs and Collège des médecins du Québec. (2016). La sédation palliative en fin de vie. http://www.cmq.org/publications-pdf/p-1-2016-08-29-fr-sedationpalliative-fin-de-vie.pdf

Tomczyk, M., \& Dieudonné-Rahm, N. (2019). La conceptualisation de la sédation palliative par les infirmiers exerçant au sein de l'unité de soins palliatifs de l'Hôpital fribourgeois, en Suisse romande. Une recherche qualitative exploratoire. Recherche en soins infirmiers, 137, 6-17.

Tong, A., Sainsbury, P., \& Craig, J. (2007). Consolidated criteria for reporting qualitative research (COREQ): A 32-item checklist for interviews and focus groups. International Journal for Quality in Health Care, 19(6), 349-57. https://doi.org/10.1093/intqhc/mzm042.

Van Campenhoudt, L., \& Quivy R. (2011). Manuel de recherche en sciences sociales. Dunod.

Verspieren, P. (1984). Face à celui qui meurt. Desclée de Brouwer.

Ziegler, S., Schmid, M., Bopp, M., Bosshard, G., \& Puhan, M. A. (2018). Continuous deep sedation until death in patients admitted to palliative care specialists and internists: A focus group study on conceptual understanding and administration in Germanspeaking Switzerland. Swiss Medical Weekly, Aug 22; 148:w14657. https://doi.org/smw.2018.14657. eCollection 2018 Aug 13.

Zuleta-Benjumea, A., Muñoz, S. M., Vélez, M. C., \& Krikorian, A. (2018). Level of knowledge, emotional impact and perception about the role of nursing professionals concerning palliative sedation. Journal of Clinical Nursing, 27(21-22), 3968-78. https:// doi.org/10.1111/jocn.14582. 